$2-1-2017$

\title{
Addition of the apical oblique projection increases the detection of acute traumatic shoulder abnormalities in adults
}

\author{
Kimberley J. Ross \\ Grant Tomkinson \\ University of North Dakota, grant.tomkinson@und.edu \\ Bonnie F. McGregor \\ Oliver C. Ayres \\ Diana Piscitelli
}

\section{How does access to this work benefit you? Let us know!}

Follow this and additional works at: https://commons.und.edu/ehb-fac

Part of the Health and Physical Education Commons

\section{Recommended Citation \\ Kimberley J. Ross, Grant Tomkinson, Bonnie F. McGregor, et al.. "Addition of the apical oblique projection increases the detection of acute traumatic shoulder abnormalities in adults" (2017). Education, Health \& Behavior Studies Faculty Publications. 22.}

https://commons.und.edu/ehb-fac/22

This Article is brought to you for free and open access by the Department of Education, Health \& Behavior Studies at UND Scholarly Commons. It has been accepted for inclusion in Education, Health \& Behavior Studies Faculty Publications by an authorized administrator of UND Scholarly Commons. For more information, please contact und.commons@library.und.edu. 


\title{
Addition of the apical oblique projection increases the detection of acute traumatic shoulder abnormalities in adults
}

Kimberley J. Ross, Grant R. Tomkinson, Bonnie F. McGregor, Oliver C. Ayres, Diana Piscitelli

\begin{abstract}
Purpose

Plain radiographic evaluation of acute shoulder trauma in adults requires a minimum of two projections, commonly the anteroposterior (AP) and lateral scapular projections, with additional projections taken for diagnosis. The aim of this retrospective study was to determine whether the addition of the apical oblique (AO) projection to the AP and lateral scapular projections increases the number and/or alters the types of abnormalities detected in the examination of acute shoulder trauma.
\end{abstract}

\section{Methods}

Examinations of 56 adults who had undergone three-projection (AP, lateral scapular, AO) radiographic shoulder examination for acute trauma were allocated into two-projection (AP, lateral scapular) and threeprojection cases and assessed by a radiologist. The differences in number and types of abnormalities between the two-projection and three-projection cases were quantified using the one-tailed $t$ test and chisquare goodness-of-fit test, respectively.

\section{Results}

Test-retest reliability was moderate (intra-class correlation coefficient [95\% CI], 0.56 [0.15 to 0.80]) for number, and almost perfect (kappa [95\% CI], 0.94 [0.85 to 1.00]) for types, of abnormalities detected. There was a significant increase in the number of abnormalities detected across all three-projection versus two-projection cases (difference in means [95\% CI], 0.20 [0.01 to 0.39]) and for fractures (difference in means [ $95 \% \mathrm{CI}], 0.30$ [0.11 to 0.49$]$ ), but no difference in the types of abnormalities detected $\left(\chi^{2}=4.7, p=0.19\right)$.

\section{Conclusion}

This study suggests that adding the AO projection to two-projection examination of acute shoulder trauma increases the number of abnormalities detected; this has potential implications for patient management. Further research investigating differences in types of abnormalities detected between twoprojection and three-projection cases is warranted.

\section{Keywords}

Apical oblique, Shoulder, Trauma, Emergency, Radiography, Projection

\section{Introduction}

Plain radiography is recognized as the first-line imaging modality for evaluating shoulder pain and trauma due to its accessibility, low cost, short examination time, clinician familiarity, and suitability for assessing a broad range of abnormalities [1,2,3]. Plain radiographic evaluation of the shoulder requires a minimum of two projections performed perpendicular to each other, typically an anteroposterior (AP) and a type of lateral scapular or axillary projection $[4,5]$. To optimize visualization of shoulder anatomy on a variety of patient presentations, several plain radiographic projections can be adopted [6]; however, there is currently no consensus in the literature regarding the diagnostic performance of the different projections. 
One of these projections is the apical oblique (AO) (Figs. 1 and 2), a modification of the axillary projection and first described by Garth et al. [7] for the detection of glenohumeral instability. Despite the AO projection being suitable for many patients, relatively simple to perform and able to be obtained rapidly [8], it is not typically performed in plain radiography shoulder protocols. To date, only two studies, which are now several decades old, have evaluated the diagnostic performance of the AO projection for the detection of acute shoulder abnormalities in adults [8, 9]. Kornguth and Salazar [8] conducted a retrospective analysis of 511 adult radiographic shoulder examinations and reported that when assessed individually, the AO projection identified slightly fewer abnormalities than the AP projection but performed better when compared with the transthoracic and lateral scapular projections. Furthermore, the AO was the only projection to detect unique abnormalities (i.e., glenoid rim and HillSachs fractures, posterior subluxation and soft tissue calcification). Richardson et al. [9] subsequently reported that as single projections, the AO projection was superior to the Neer lateral (i.e., lateral scapular) projection at detecting true positive and true negative fractures and dislocations in a prospective analysis of 244 adults. These two studies [8,9] highlight that the AO projection may be beneficial as a complementary projection to the AP and lateral scapular or axillary projection in the evaluation of acute shoulder trauma.

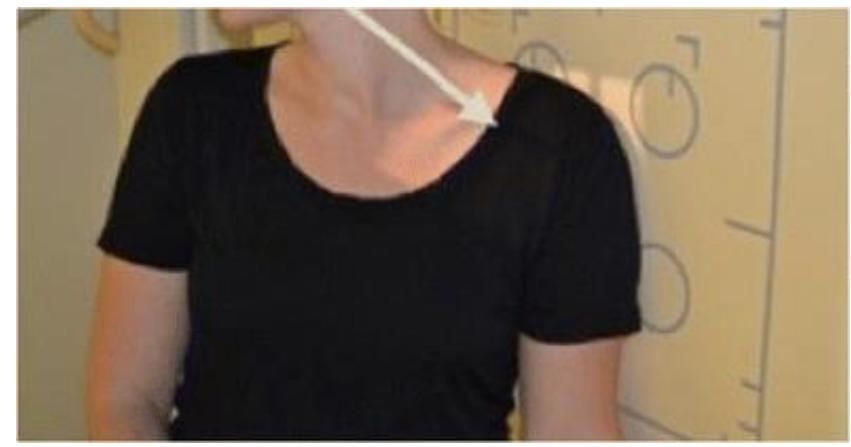

Fig. 1

Patient positioning demonstrating the centering point and direction of the central ray for the apical oblique (AO) projection

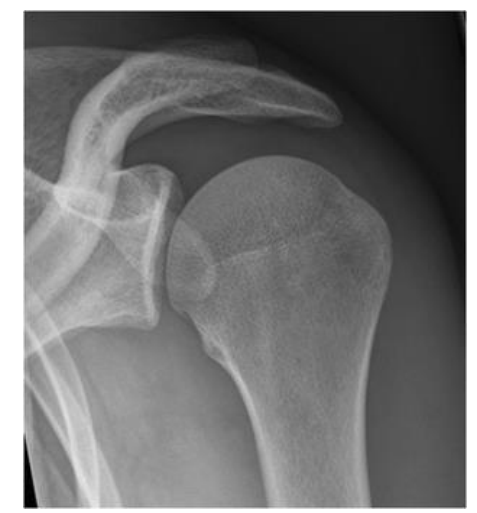

Fig. 2

Plain radiographic appearance of the apical oblique (AO) projection

As a result of poor visualization of the bony structures of the glenohumeral joint in the lateral scapular projection and difficulties in positioning acutely injured patients for the axillary projection, the Department of Medical Imaging, Flinders Medical Centre, Bedford Park, Australia, introduced the AO projection as an additional third projection to the acute plain radiographic shoulder protocol. Therefore, the aim of this study was to evaluate this new protocol by determining whether the addition of the AO projection to the routinely performed AP and lateral scapular projections increases the number and/or 
alters the types of abnormalities detected in the examination of acute shoulder trauma in adults. The results of this study have the potential to inform and change current clinical radiographic practice by establishing whether the AO projection is a beneficial complementary projection for the detection of acute shoulder abnormalities.

\section{Materials and methods Ethical approval and informed consent}

This study employed a retrospective, non-experimental, quantitative research design. Ethics approval was granted by the Southern Adelaide Clinical (protocol number, 327.14) and the University of South Australia (protocol number, 0000033612) Human Research Ethics Committees. Informed consent was waived due to the retrospective design of this study and in accordance with the National Statement on Ethical Conduct in Human Research [10].

\section{Patients}

The Picture Archiving and Communication System in the Department of Medical Imaging, Flinders Medical Centre, Bedford Park, Australia, was searched using the keywords shoulder and humerus to identify patients who fulfilled the selection criteria. Using consecutive sampling, patient examinations were included in the study if three projections (i.e., AP, lateral scapular, and AO) were performed to investigate formal medical referrals for acute shoulder trauma on patients aged $\geq 18$ years with at least one acute traumatic abnormality (e.g., humeral head fracture, glenoid fracture, or humeral head dislocation) detected by the radiologist who originally reported the examination. Exclusion criteria were (a) investigation of chronic conditions, (b) focus specific to the thorax, clavicle or acromioclavicular joints, (c) reference to previous examinations required on the same patient for reporting purposes (excluding the clinical indication of post-reduction), and (d) examinations not of diagnostic quality.

Power calculations using a repeated measures design on a two-tailed $\mathrm{F}$ test with four categories (i.e., four shoulder trauma categories) determined that to detect a difference in the number of abnormalities between two-projection and three-projection cases, 56 patient examinations were required to provide an $80 \%$ power, at the 0.05 significance level. Due to the potential to change patient management, clinical relevance was defined as the detection of just one extra abnormality using the AO projection.

\section{Protocol \\ Data collection and organization}

Between April 2012 and October 2013, 56 of 1750 (3.2\%) shoulder and humerus examinations performed met the inclusion criteria (32 males, 24 females, aged 18-97 years). The remaining patient examinations were excluded due to medical referrals for unrelated clinical indications, radiographer compliance with the newly implemented protocol, and if an alternative projection was performed (e.g., axial). These 56 digital patient examinations, excluding the clinical information, were downloaded, de-identified, assigned a patient number from 1 to 56, and then copied to form two groups. The three-projection group consisted of 56 cases each containing three projections (labelled $1 \mathrm{~b}$ to $56 \mathrm{~b}$ ), and the two-projection group consisted of a copy of the 56 cases with the AO projection removed (labelled 1a to 56a).

\section{Radiologist reporting procedure}

A radiologist who is a fellow of the Royal Australian and New Zealand College of Radiologists (FRANZCR) with 8 years of experience randomly viewed and assessed all patient cases at two time points, separated by 3 months in order to minimize memory effect. The radiologist was blinded to the study design including the inclusion and exclusion criteria. 
At time-point 1, cases $1 \mathrm{a}$ to $32 \mathrm{a}$ and $33 \mathrm{~b}$ to $56 \mathrm{~b}$ were examined, with 10 cases (i.e., $5 \times 2$-projection and 5 $\times 3$-projection cases) chosen at random and duplicated to allow for the determination of intra-rater reliability. The 66 cases were then allocated a new case number from 1 to 66 . The radiologist recorded the number and type of acute abnormalities detected for each case and provided a brief radiological report. The number of abnormalities detected was defined as the total number of abnormalities identified on each radiographic projection by the radiologist.

At time-point 2 (i.e., 3 months after time-point 1), the original two-projection cases with the corresponding $\mathrm{AO}$ projection (cases $1 \mathrm{~b}$ to $32 \mathrm{~b}$ ), the original three-projection cases without the corresponding AO projection (cases 33a to 56a), and the same subset of ten duplicate cases from timepoint 1 (i.e., with the $\mathrm{AO}$ projection added to the two-projection cases and $\mathrm{AO}$ projection removed from the three-projection cases [i.e., 66 cases]) were allocated a new case number from 67 to 132. These cases were then viewed and assessed by the radiologist, recording information as per time-point 1 .

\section{Statistical analysis}

All data provided by the radiologist were entered into an Excel spreadsheet by the chief investigator and checked by a co-investigator for statistical analysis in XLSTAT (Addinsoft, New York, USA).

The intra-rater reliability of the radiologist was assessed using the test-retest data in two ways. First, the reliability of the number of abnormalities detected was assessed by the intra-class correlation coefficient (ICC). Second, the reliability of the types of abnormalities detected was assessed by the kappa coefficient when classified into four acute shoulder trauma categories: none, fractures, dislocations/subluxations, and soft tissue injuries. These four categories were chosen to encompass all possible acute traumatic shoulder abnormalities. In the event that the radiologist detected one abnormality in each of two different categories, they were recorded and considered as two separate abnormalities. Kappa values were qualitatively interpreted using the benchmark scale for kappa recommended by Landis and Koch [11]. Values of $<0.00$ indicated poor agreement; $0.00-0.20$, slight agreement; $0.21-0.40$, fair agreement; $0.41-$ 0.60 , moderate agreement; $0.61-0.80$, substantial agreement; and 0.81-1.00, almost perfect agreement [11].

The difference in the mean number of abnormalities between the two-projection and three-projection cases was quantified using a one-tailed $t$ test for all cases and for each shoulder trauma category separately, with probabilities corrected using sequential Bonferroni adjustments. Differences in means were calculated as absolute differences and standardized effect sizes, with positive differences indicating that more abnormalities were detected from three-projection cases, and standardized effect sizes qualitatively interpreted using thresholds of $0.2,0.5$, and 0.8 for small, moderate, and large, respectively [12]. Differences in the types of abnormalities (i.e., the four shoulder trauma categories) between the twoprojection and three-projection cases was quantified using a chi-square goodness-of-fit test. The criterion of significance was set at 0.05 .

\section{Results}

\section{Intra-rater reliability Number and type}

The test-retest reliability of the number of detected abnormalities was moderate (ICC [95\%CI] 0.56 [0.15 to 0.80$]$ ); the reliability of the type of detected abnormalities was almost perfect (kappa [95\% CI] 0.94 [0.85 to 1.00$]$ ). 


\section{Differences in shoulder abnormalities detected between two-projection and three-projection cases Number}

There was a statistically significant increase in the number of acute shoulder abnormalities detected when comparing all three-projection versus two-projection cases (difference in means [95\% CI], 0.20 [0.01 to 0.39]; fold change, 1.1; effect size, small). Conversely, Table 1 demonstrates that this was not true for all shoulder trauma categories (fractures, dislocations, subluxations, and soft tissue injuries), with the number of detected abnormalities only significantly different (larger) for three-projection versus two-projection cases for fractures (difference in means [95\% CI], 0.30 [0.11 to 0.49]; fold change, 1.3; effect size, small). Table 1 also depicts that the most frequent difference was zero (i.e., the same number of abnormalities was detected) and that there was considerable variability in the frequency of differences that were not zero, ranging from $2 \%(1 / 56)$ for dislocations to $48 \%$ (27/56) for fractures.

Table 1

Frequency distribution of the differences in the number of two-projection- and three-projection-detected abnormalities per patient across all shoulder trauma categories

\begin{tabular}{|l|l|l|l|l|}
\hline Difference & Fractures & Subluxations & Dislocations & Soft tissue \\
\hline+2 & 2 & & & \\
\hline+1 & 19 & 2 & & \\
\hline 0 & 29 & 52 & 55 & 51 \\
\hline-1 & 6 & 2 & 1 & 5 \\
\hline${\text { Mean }[95 \% \mathrm{CI}]^{\mathrm{a}}}^{\mathrm{n}}$ & $0.30[0.11 \text { to } 0.49]^{\mathrm{b}}$ & $0.00[-0.07$ to 0.07$]$ & $-0.02[-0.06$ to 0.02$]$ & $-0.09[-0.17$ to -0.01$]$ \\
\hline
\end{tabular}

Differences in the number of abnormalities detected per patient were calculated by subtracting the number of two-projection-detected abnormalities from the number of three-projection-detected abnormalities, with positive differences indicating that more abnormalities were detected from three-projection cases

${ }^{a}$ Mean differences and the corresponding $95 \%$ confidence intervals $(95 \% \mathrm{CI})$ are shown in the bottom row

${ }^{\mathrm{b} B o n f e r r o n i-c o r r e c t e d ~ s i g n i f i c a n t ~ d i f f e r e n c e s ~}$

Further examination of the fracture data suggests that three-projection cases were better than twoprojection cases at detecting particular types of fractures (Fig. 3). Table 2 presents the number of twoprojection- and three-projection-detected fractures categorized into ten fracture subcategories. These data reveal that the three-projection cases were better than two-projection cases at identifying fractures of the articular anatomical structures of the shoulder joint, with a greater number of glenoid (2.5-fold), humeral head (1.5-fold), Hill-Sachs (1.4-fold), and other fractures (1.4-fold) identified. Other fractures included, but were not limited to, greater tubercle and acromion fractures.
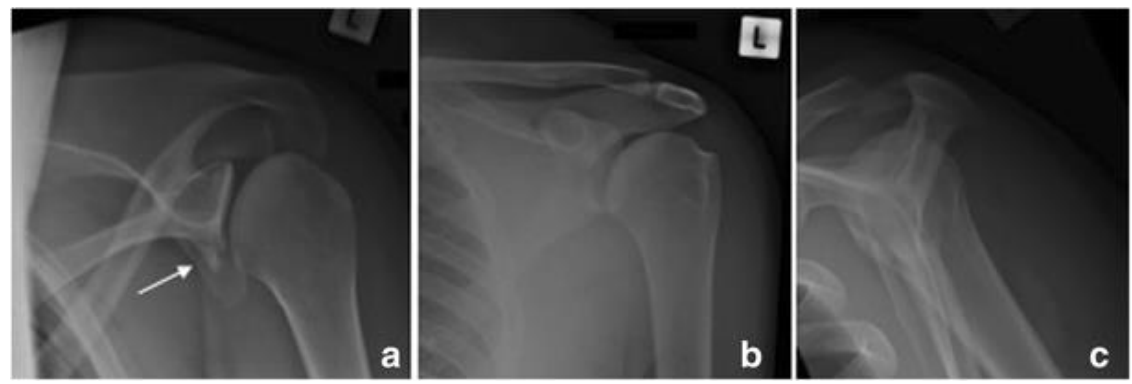

Fig. 3

Addition of the a apical oblique (AO) projection to the $\mathbf{b}$ anteroposterior (AP) and clateral scapular projections identified a Bankart fracture (arrow)

Table 2

The total number of two-projection- and three-projection-detected fractures

\begin{tabular}{|l|l|l|l|l|}
\hline \multirow{2}{*}{ Fracture } & Two projections & Three projections & \multicolumn{2}{|l|}{ Difference } \\
\cline { 2 - 6 } & $\boldsymbol{n}$ & $\boldsymbol{n}$ & $\boldsymbol{n}$ & Fold change \\
\hline Glenoid & 4 & 10 & 6 & 2.5 \\
\hline Hill-Sachs & 14 & 19 & 5 & 1.4 \\
\hline
\end{tabular}




\section{Type}

\begin{tabular}{|l|l|l|l|l|}
\hline Other & 12 & 17 & 5 & 1.4 \\
\hline Humeral head & 4 & 6 & 2 & 1.5 \\
\hline Bankart & 3 & 4 & 1 & 1.3 \\
\hline Scapular & 2 & 3 & 1 & 1.5 \\
\hline Humerus & 1 & 1 & 0 & 1.0 \\
\hline Surgical neck & 12 & 12 & 0 & 1.0 \\
\hline Clavicle & 7 & 5 & -2 & 0.7 \\
\hline Anatomical neck & 2 & 0 & -2 & 0.0 \\
\hline
\end{tabular}

There was no statistically significant difference in the types of abnormalities detected between twoprojection and three-projection cases $\left(\chi^{2}=4.7, p=0.19\right)$.

\section{Discussion}

This study is the first to evaluate the diagnostic performance of the addition of the AO projection to the routinely performed AP and lateral scapular projections in the examination of acute shoulder trauma in adults. The results of this study demonstrate that in this group, there is a significant increase in the number but not the type of abnormalities detected when the three projections are assessed concurrently. The increase in the number of detected abnormalities highlights the capacity of the AO projection to add diagnostic information in routine clinical practice and, hence, has potential implications for patient management.

Early research by Kornguth and Salazar [8] demonstrated in patients referred for acute shoulder trauma that when assessed as single projections, the AO projection detected $81 \%$ of all fractures, dislocations, subluxations, calcifications, and foreign bodies slightly (0.9-fold) worse than the AP projection which detected $88 \%$, yet substantially (1.5- to 1.8 -fold) better than the transthoracic and lateral scapular projections which detected 44 and 55\%, respectively. In spite of the differing study designs between this and the previous [8] study (i.e., assessment of single versus multiple projections), our findings are supported by those of Kornguth and Salazar [8]. Also, consistent with our findings, Richardson et al. [9] reported a substantially greater number of fractures and dislocations detected by the AO, compared with the lateral scapular, projection. We found that the addition of the AO projection to the AP and lateral scapular projections led to a small increase in the number of detected fractures ( $n=16$ or 1.3-fold), with a greater number of Hill-Sachs (1.4-fold), humeral head (1.5-fold), glenoid (2.5-fold), and other(1.4-fold) fractures reported. While Kornguth and Salazar [8] also found the AO projection to be the most sensitive of the four projections at detecting Hill-Sachs and glenoid rim fractures, their data were not quantified statistically. Therefore, these findings should be considered as suggestive only. Our study failed to observe a statistically significant difference in the types of abnormalities detected between two-projection and three-projection cases, which may be due to the sample size used in this study.

While the main strengths of this study include that a sound methodology was utilized that reflected current clinical practice and projections were obtained with contemporary medical imaging equipment, it is important to point out its limitations. Although adequately powered to detect the observed differences in the number of abnormalities, this study was underpowered to detect the observed difference in the types of abnormalities. In a minority of cases, there was a greater number of abnormalities detected with two projections versus three projections. Moreover, despite three-projection cases being overall better than two-projection cases at detecting fractures, individual data for anatomical neck and clavicle fractures were in contrast to this finding. Both observations may be indicative of some degree of intra-rater variability. While the results of this study indicate that the ability (of at least one experienced radiologist) to reliably assess acute shoulder abnormalities was moderate (for number) to almost perfect (for type), it is not known how well the assessments made by a single radiologist compare with those of other 
radiologists; the inclusion of at least two radiologists to assess inter-rater reliability should be considered in future study design. This study also did not include an assessment of the radiation dose, financial cost, and time burden associated with increasing the number of routine projections. Despite these limitations, the results of the present study provide support for the AO projection to be included as routine clinical practice (at least in terms of the ability of three projections to add diagnostic information through the detection of an increased number of acute shoulder abnormalities). It should be noted, however, that while detection of just one additional abnormality has the potential to change patient management and therapeutic intervention, this may not always be the case (e.g., glenoid fracture versus a minor Hill-Sachs lesion) and future research should explore this further. Furthermore, although substantial patients undergo $\mathrm{CT}$ and/or MRI examinations in addition to plain radiography to aid treatment planning, adopting a threeprojection protocol may reduce the use of these imaging modalities, depending on the radiologist's initial assessment. Our findings may also lead to change in clinical practice in environments where CT and/or MRI are less easily accessible and further research in this area is indicated. Lastly, this study highlights the need to determine which combination and number of projections result in optimal diagnostic performance for the diagnosis of acute shoulder trauma.

\section{Conclusion}

This study establishes that the addition of the AO projection, to the AP and lateral scapular projections, in the examination of acute shoulder trauma in adults increases the number of abnormalities detected and, therefore, provides support for a review of current radiographic clinical practice. Furthermore, given that when assessed together, the three projections result in a greater number of fractures being detected; the findings of this study have potential implications for the therapeutic management of these patients.

\section{Notes}

\section{Acknowledgments}

The authors would like to acknowledge the Department of Medical Imaging, Flinders Medical Centre, Bedford Park, Australia, for their support in providing the patient examinations that were utilized in the conduct of this study. Data from this manuscript have been presented in abstract form at the 11th Annual Scientific Meeting of Medical Imaging and Radiation Therapy, Brisbane, Australia, 22 April 2016 (Ross KJ, Tomkinson GR, McGregor BF, Ayres OC, Piscitelli, D (2016) The effectiveness of the addition of the apical oblique projection in the detection of acute shoulder abnormalities in adults. JMRS 63(Supp S1):42).

\section{Compliance with ethical standards}

\section{Conflict of interest}

The authors declare that they have no conflict of interest.

\section{Ethical approval}

This retrospective study was approved by institutional ethics committees, and the need to obtain informed consent was waived.

\section{References}

1. Koh KH, Han KY, Yoon YC, Lee SW, Yoo JC (2013) True anteroposterior (Grashey) view as a screening radiograph for further imaging study in rotator cuff tear. J Shoulder Elb Surg 22(7): 901-907 
2. Bianchi S, Prato N, Martinoli C, Derchi LE (2006) Shoulder radiography. In: Davies AM, Holder J (eds) Imaging of the shoulder: techniques and applications. Springer, Berlin, pp 3-13

3. Cadogan A, Laslett M, Hing WA, McNair PJ, Coates MH (2011) A prospective study of shoulder pain in primary care: prevalence of imaged pathology and response to guided diagnostic blocks. BMC Musculoskelet Disord. doi:10.1186/1471-2474-12-119

4. Jensen KL, Rockwood Jr CA (2009) Radiographic evaluation of shoulder problems. In: Rockwood Jr CA, Matsen 3rd FA (eds) The shoulder. Saunders Elsevier, Philadelphia, pp 177-212

5. Neep MJ, Aziz A (2011) Radiography of the acutely injured shoulder. Radiography 17(3):188-192

6. Sanders TG, Jersey SL (2005) Conventional radiography of the shoulder. Semin Roentgenol 40(3):207-222

7. Garth WP Jr, Slappey CE, Ochs CW (1984) Roentgenographic demonstration of instability of the shoulder: the apical oblique projection. A technical note. J Bone Joint Surg Am 66(9):1450-1453

8. Kornguth PJ, Salazar AM (1987) The apical oblique view of the shoulder: its usefulness in acute trauma. Am J Roentgenol 149(1): 113-116

9. Richardson JB, Ramsay A, Davidson JK, Kelly IG (1988) Radiographs in shoulder trauma. J Bone Joint Surg Br 70(3):457-460

10. National Health and Medical Research Council (Australia), Australian Research Council, Australian Vice-Chancellors' Committee (2007) National statement on ethical conduct in human research E72. National Health and Medical Research Council, Canberra

11. Landis JR, Koch GG (1977) The measurement of observer agreement for categorical data. Biometrics 33(1):159-174

12. Cohen J (1988) Statistical power analysis for the behavioral sciences, 2nd edn. Lawrence Erlbaum Associates, Hillsdale, New Jersey 